\title{
Sperm-limited males save ejaculates for future matings when competing with superior rivals
}

Dolores Schütz ${ }^{1}$, Linda Tschirren ${ }^{1}$, Gudrun Pachler ${ }^{1}$, Pia Grubbauer ${ }^{1} \&$ Michael Taborsky $^{1, *}$ 


\section{Abstract}

Ejaculate adjustments to sperm competition can lead to sperm limitation. Particularly in polygynous species, males may face a trade-off between investing sperm in current or future mating opportunities. The optimal sperm allocation decision should depend on the relative intensity of

6 sperm competition experienced in a mating sequence. Here we ask how males respond to this trade-

7 off in polygynous fish with alternative male mating tactics, intense sperm competition and sperm

8 limitation. Large bourgeois males of the shell brooding cichlid Lamprologus callipterus build nests

9 consisting of empty snail shells, in which females spawn and raise offspring. During spawning, nest

10 males release ejaculates into the shell opening. Genetically distinct, parasitic dwarf males enter

11 shells during spawning to fertilize the eggs from inside the shell. These dwarf males were

12 previously shown to be superior sperm competitors to nest males. Here we show that when

13 spawning with several females simultaneously, nest males reduce the spawning duration for each

14 clutch and the number of ejaculations per female tends to decrease, reflecting sperm limitation.

15 Experimental exposure of nest males to sperm competition with dwarf males caused a reduction of 16 the number and duration of ejaculations roughly by half. Hence, when exposed to competition with 17 a superior rival, nest males do not increase their sperm expenditure as predicted by sperm

18 competition risk models, but in fact save sperm for future mating opportunities as predicted by 19 sperm competition intensity theory. This seems to be adaptive because of the considerable sperm 20 demands in this species, which is partly due to their high degree of polygyny.

Keywords: alternative mating tactics, bourgeois and parasitic males, ejaculate allocation, sperm competition, sperm depletion 
Sperm competition, where sperm of two or more males compete for the fertilisation of eggs (Parker, 1970), can cause behavioural and morphological adaptations in males. Adaptive adjustment to sperm competition includes prolonged copulations (Schöfl \& Taborsky, 2002), mate guarding and an increase in copulation frequency (Birkhead, 1998), sperm displacement (Parker \& Simmons, 1994), the development of copulatory plugs (Dunham \& Rudolf, 2009), and breakage of copulatory organs (Snow, Abdel-Mesih, \& Andrade, 2006). Males may monopolise either limited resources for breeding or females directly to prevent rival males from gaining access to females (bourgeois tactic; Taborsky, 1997; Taborsky \& Brockmann, 2010). As a consequence of such mating monopolisation, male reproductive success is typically strongly skewed, and alternative reproductive tactics (ARTs; Oliveira, Taborsky, \& Brockmann, 2008) may evolve, where parasitic males invest relatively more in testes than bourgeois males due to their higher risk of sperm competition ( Byrne, Roberts, \& Simmons, 2002; Stockley, Gage, Parker, \& Møller, 1997; Taborsky, 1998). received less empirical support (for reviews see Engqvist \& Reinhold, 2005; Kelly \& Jennions,

51 2011; Wedell et al., 2002). These models imply that generally speaking, if males can succeed in 
sperm competition they should increase ejaculate expenditure, whereas if they have little chances to succeed against superior competitors, they should reduce ejaculate expenditure to save sperm for future matings. This may be particularly important in polygynous species, where both mating frequency and sperm competition can lead to sperm limitation (Wedell et al., 2002). Additional causes of sperm limitation (Shapiro \& Giraldeau, 1996) include low fertilization efficiency, large clutch size, and high reproductive costs for males (Wedell et al., 2002).

It is generally little understood to which extent variation in sperm and ejaculation characteristics result from either sperm competition or sperm limitation. In bitterling fish, for example, differences in the mating system (Pateman-Jones et al., 2011) or breeding resource distribution (Reichard, Ondrackova, Bryjova, Smith, \& Bryja, 2009) significantly affect ejaculate characteristics under high levels of both sperm competition and sperm limitation. An interspecific comparison showed that the species with the shortest spawning season (Rhodeus amarus), which corresponds to a high probability of sperm limitation, showed the greatest level of investment in sperm production, the highest ejaculation rate, the smallest clutch size, and spermatozoa apparently adapted to fast swimming (Pateman-Jones et al., 2011). With a clumped breeding resource distribution in comparison to an even resource distribution, stronger selection on traits that evolve due to sperm competition was detected (Reichard et al., 2009). In sea urchins, high population densities result in selection on sperm traits associated with sperm competition, whereas low population densities result in selection on sperm traits associated with sperm limitation (Levitan, 2002).

A possibility to disentangle the influence of these two challenges on sperm and ejaculate characteristics is to manipulate the degrees of sperm competition and polygyny independently of each other in species that combine polygyny with the existence of ARTs. To test for an influence of sperm limitation, it can be determined how males adjust ejaculation characteristics to the number of 
females they mate with at a time. To test for an influence of sperm competition, ejaculation characteristics can be compared between bourgeois males mating alone or together with parasitic males. However, in externally fertilising species releasing their gametes into the water, it is difficult to determine ejaculation characteristics under different experimental conditions (Shapiro, Marconato, \& Yoshikawa, 1994).

In the cichlid fish Lamprologus callipterus, large bourgeois males construct and defend nests consisting of empty snail shells, in which the much smaller females breed (further on called 'nest males'; Schütz \& Taborsky, 2005). Females lay clutches containing on average 95 eggs, which are deposited one by one inside a shell with intervals of more than two minutes between subsequent eggs (Schütz, Heg-Bachar, Taborsky, \& Heg, 2012). Each egg needs to be fertilized by a separate ejaculate, leading to a total spawning duration of nearly seven $\mathrm{h}$ on average (Schütz et al., 2012) and to severe sperm limitation of nest males (Schütz, Pachler, Ripmeester, Goffinet, \& Taborsky, 2010). The latter reduce the number of sperm released per ejaculate drastically after $5 \mathrm{~h}$ of continuous spawning, even when spawning with only one female (Schütz et al., 2010), and even though eggs are deposited at a similar rate over the whole duration of a spawning (Schütz et al., 2012).

In L. callipterus, two distinct parasitic male types exist: Sneaker males opportunistically try to enter territories where nest males are spawning to fertilize eggs by releasing ejaculates into the shell opening when the nest male is inattentive. This tactic is transitional and performed by males of the nest male type (Schütz, Parker, Taborsky, \& Sato, 2006). Parasitic dwarf males constitute a genetically distinct male morph (Wirtz Ocana, Meidl, Bonfils, \& Taborsky, 2014) remaining small throughout life (Taborsky, 2001). They try to enter shells in which a female is spawning to fertilize the eggs from inside the shell. Once succeeded, dwarf males stay in the shell during the whole spawning event, and therefore they are in much closer vicinity to the female and eggs during laying 
102

103

104

105

106

107

108

109

110

111

112

113

114

115

116

117

118

119

120

121

122

123

than nest males, i.e. they are in a privileged position to fertilise the eggs (Sato, Hirose, Taborsky, \&

Kimura, 2004). This is in contrast to most other species, where bourgeois males are usually much closer to eggs than parasitic males (Taborsky, 2008; Taborsky, Oliveira, \& Brockmann, 2008).

Thus, spawning of a nest male with a parasitic dwarf male resembles a loaded raffle (Parker, 1990a)

where dwarf males have a fertilization advantage, which is revealed also by the much greater fertilization success of dwarf males in comparison to nest males in nature (Wirtz Ocana et al., 2014). Theory predicts that the unprivileged male type (here the nest male) should compensate his disadvantage by investing more in the present ejaculate than the privileged male type (here the dwarf male; Parker, 1990a). Hence, in accordance with sperm competition risk models nest males should increase their reproductive effort when spawning with a parasitic dwarf male (Parker, 1998). Alternatively, nest males might decrease ejaculate expenditure in such competitive situations to save sperm for future spawning opportunities without participating dwarf males, as predicted by sperm competition intensity models especially if they involve highly loaded raffles (Parker et al., 1996, Parker et al., 1997).

In species with external fertilisation, ejaculate sizes can hardly be determined exactly because sperm diffuse into water right after release. This is different in L. callipterus, since males ejaculate into a snail shell, which allows collection of their sperm and determination of ejaculation characteristics. Additionally, the perceived risk of sperm competition for nest males can be manipulated directly by adding a dwarf male into a shell where the nest male is spawning. Thus, physiological responses of males to the perceived risk of sperm competition before a test spawning are prevented, avoiding this pitfall in testing predictions from sperm competition models (Engqvist \& Reinhold, 2005).

Here we aim to clarify the relative roles of sperm limitation and sperm competition for shaping 
127 ejaculation characteristics of L. callipterus nest males. Specifically, we ask how much they invest in

128 ejaculates in dependence of increasing sperm limitation and sperm competition risks. We compared

129 nest male sperm and ejaculation characteristics when spawning (i) with one or more females

130 simultaneously to test for the influence of sperm limitation, and (ii) with or without a parasitic

131 dwarf male present to test for the influence of sperm competition.

133 We investigated how nest males deal with the apparent trade-off regarding sperm allocation in 134 dependence of the current sperm competition risk and future mating opportunities (Wedell et al., 135 2002). When facing sperm competition with a superior dwarf male, nest males may either increase 136 ejaculate expenditure to compensate for their `devalued` fertilisation opportunity (Parker, 1990b; 137 Parker, 1998) or decrease it to save sperm for future matings without dwarf males (cf. Parker et al., 138 1996, Parker et al., 1997, Wedell et al., 2002). In the laboratory, we experimentally added a dwarf 139 male into a shell where a female was spawning with a nest male and analysed nest male behaviour 140 and ejaculate characteristics before and after this manipulation.

142 To estimate the risk of sperm competition and sperm limitation in the natural situation, we 143 determined the number of females, intruders of the nest male type (mainly territorial neighbours), 144 sneakers, and dwarf males entering a nest per day from long-term video recordings obtained in the 145 field. Sperm limitation of nest males should be even higher when nest males spawn with more than 146 one female at a time. Thus, from these long-term video recordings we determined the total 147 spawning duration for each clutch and the number of ejaculations per female for nest males that 148 were spawning simultaneously with different numbers of females.

150 In addition, we determined the relationship between ejaculation characteristics and sperm numbers 151 in laboratory experiments in which nest males spawned with a female alone, since it is impossible 
152 to determine sperm numbers of nest males reliably when dwarf males participate in spawning. We 153 video-taped nest male ejaculations, collected and counted their sperm, and tested the relationship 154 between different ejaculation characteristics. We determined the number of sperm per ejaculation, 155 the duration of each ejaculation bout, and the sperm release rate (sperm number per ejaculate / 156 duration of ejaculation bout), and tested how these parameters relate to the total number of sperm 157 released during the entire spawning.

\section{Methods}

Study species

L. callipterus is a cichlid fish endemic to Lake Tanganyika, which shows the greatest sexual size dimorphism among animals with males being bigger than females (Schütz \& Taborsky, 2000). Two genetically determined alternative reproductive tactics exist: (i) Nest males: after passing a size threshold of $9 \mathrm{~cm}$ standard length (Schütz \& Taborsky, 2005), these males build and defend nests consisting of clumps of empty snail shells, in which females lay eggs and care for the brood for 10 to 14 days (Sato, 1994; Sato et al., 2004; Schütz \& Taborsky, 2000). Nest males collect empty snail

169 shells from the vicinity of their nests or steal them from neighbouring males (Mitchell, Wirtz

170 Ocana, \& Taborsky 2014; Sato, 1994). Since nest males hardly feed when defending a territory, 171 their condition decreases during territory maintenance until their territories are taken over by larger 172 or heavier males (Sato, 1994; Schütz et al., 2010). Males pursuing this life history pathway may 173 adopt also a parasitic mating tactic, particularly when they are still too small to successfully defend 174 a nest. Such 'sneaker' males try to enter a nest owner's territory during spawning to fertilize eggs 175 by releasing ejaculates into the shell opening (Sato et al., 2004). (ii) Dwarf males: These males remain even smaller than females throughout life (Taborsky, 2001; Wirtz Ocana et al., 2014); in the 
177 field, they weigh on average only $2.5 \%$ of nest males (Sato et al., 2004). Dwarf males try to enter a 178 shell with a spawning female inside by wriggling past the female in order to enter the inner whorl of 179 the shell, from where they can fertilize the eggs (Sato et al., 2004). If successful in their attempt to 180 enter the shell, they fertilize the majority of the eggs from this privileged position (Wirtz Ocana et 181 al., 2014).

183 Holding conditions for fish in the laboratory

All fish used in laboratory experiments were offspring of wild-caught fish originating from the southern end of Lake Tanganyika, Zambia. They were fed twice per day on 6 days of the week, with protein-rich food (frozen zooplankton) in the morning and with flake food in the evening. Water parameters were kept close to the values known from the Southern end of Lake Tanganyika. Light was kept at a 13:11 light:dark cycle with 10-min dimmed light phases in between. All tanks were equipped with a biological filter.

$\underline{\text { Female visits and sperm competition in nature }}$

To check for the potential risk of sperm competition and sperm limitation, we determined how many females and males of different tactics are found around territories at Lake Tanganyika. As the spawning activity in this species varies significantly with the lunar cycle (Nakai, Yanagisawa, Sato,

197 Niimura, \& Gashagaza, 1990), we attempted to monitor nests throughout the different phases of the 198 moon cycle. During two field seasons, seven nests at Wonzye Point (1995) and four nests at 199 Kasakalawe Point (1997) were continuously recorded via underwater video-cameras from new 200 moon to one week after full moon. In ten of these nests, spawning was recorded. All videos were 201 analysed for the entire daylight period (6:00h-18:00h). From these video recordings, we determined 
the number of females, nest male intruders (mainly neighbouring males that try to steal shells or to take over the nest), sneaker males and dwarf males (males trying to steal fertilizations) entering a nest per day, and we calculated averages per moon day.

We divided the moon cycle into 3 day intervals ( 7 intervals for 3 weeks of recording) and checked whether the frequency of dwarf plus sneaker males, nest male intruders and visiting females differed from equal distributions with $\mathrm{Chi}^{2}$-tests. Additionally, we used $\mathrm{Chi}^{2}$-tests to check whether the frequency of dwarf plus sneaker males and of nest male intruders differed from the frequency of females visiting a nest per day. For statistical analyses, we used the software package IBM SPSS

211 statistics 22 and tested for two-tailed probabilities. For the LMM of sperm number per ejaculation

212 in dependence of time of sperm release we used the package "Ime4" of the software R 3.2.2. (R

213 Core Team 2015).

$\underline{\text { Adjustment of ejaculations to spawning with multiple females }}$

To find out how L. callipterus nest males adjust ejaculation characteristics when spawning with more than one female at a time, we determined the total spawning duration of nest males per clutch 219 and the number of ejaculations per female when they were spawning with up to four females simultaneously. The number of ejaculations is representative for the total number of sperm if 1) these two variables correlate positively with each other, and 2) the number of sperm per ejaculation does not change significantly with time. We checked and verified these two assumptions with data from the first laboratory experiment (see below).

From continuous video recordings in the field (see above), we could identify the exact start and end of laying a clutch for 28 individual females spawning in different shells (each female spawns in a 
separate shell in this species; see Schütz et al., 2012 for details and methods). To check whether the two variables differ between males spawning with 1,2,3, or 4 females at a time, we used general linear models (GLM) with the number of simultaneously spawning females as fixed factor, and male ID as random factor. Additionally, with Spearman rank correlation analyses we tested whether the two variables relate to the number of females with which a nest male was concurrently spawning.

\section{$\underline{\text { Sperm release rate versus the duration of ejaculations }}$}

We aimed to check whether ejaculation characteristics are a reliable measure for the numbers of sperm released during a whole spawning event by counting sperm numbers of nest males when spawning alone with a female and relating these to ejaculation characteristics. These experiments were carried out in 400 litre tanks divided into two differently-sized (1:2) compartments with a clear Plexiglas partition. The bigger compartment was set up as a territory for a nest male. Five manipulated shells were secured in a position that enabled us to videotape any activity in front of them. Eight males between 8.3 and $9.9 \mathrm{~cm}$ standard length were investigated with groups of four females each. Before starting the experiment we put the four females in the large compartment (containing the shells) and the territorial male in the small compartment, so that he could see the females but could not physically interact with them until they were ready to spawn.

When a female was ready to spawn (showing an extended whitish belly), the experiment started at 9:00 a.m. on the following morning. The other 3 females were taken out of the compartment, the male was transferred from the small into the large compartment, and continuous video recording was started. To collect the sperm released by the male, we prepared shells by attaching a silicone tube (inside diameter $=2 \mathrm{~mm}$ ) to a hole drilled into the first spiral of the shell, where the eggs are 
usually placed during spawning. After each ejaculation the sperm was sucked out with a motor pump and all samples were then analysed for the presence of sperm using the methods of Leong (1989) and Shapiro et al. (1994) modified to our needs (see below).

Water samples of $30 \mathrm{ml}$ each were sucked out from the shell via the tube when the male positioned his genital papilla over the opening of the shell and supposedly released sperm (cf. Schütz et al., 2012). The amount of water contained in the tube itself was $5 \mathrm{ml}$, and the average shell-volume was $15.4 \pm 0.84 \mathrm{ml}$ (mean \pm standard deviation). We took $30 \mathrm{ml}$ for each water-sample to ensure that the tube plus shell volumes were collected completely for each extraction. The water sample was immediately mixed with the same amount of $0.1 \mathrm{M}$ phosphate buffer to eliminate the effect of osmotic pressure on sperm heads. To analyse our samples for the presence of sperm, we modified the methods of Leong (1989) and Shapiro et al. (1994) as follows. After removal, two drops of Rose Bengal were added for staining the head of the spermatozoa before passing the sample through a Millipore filter $(0.22 \mu \mathrm{m}$ pore size $)$ under vacuum. The filter paper was dried and cleared with immersion oil. The number of sperm was counted under a light microscope at a magnification of 400x in an area of $0.185 \times 0.185 \mathrm{~mm}$, and the count was repeated 20 times at randomly selected portions of the filter. The mean value of these counts was used to estimate the total number of sperm present in the sample (total filter area $=160.61 \mathrm{~mm}^{2}$, therefore the sperm count was multiplied by 4692.65).

Water samples of ejaculates were taken at intervals of about 10 minutes, and one sample from every half-hour was analysed as described above. We determined the numbers of sperm per ejaculation and the total numbers of sperm released for each test male. For each ejaculate of the analysed watersamples, from the video recordings we measured the exact duration of the ejaculation bout, i.e. how long the male placed his genital papilla above the shell entrance and calculated the ejaculation rate 
277 (ejaculations/min) for each male. The number of sperm per ejaculate divided by the duration of 278 ejaculation bouts gave the mean sperm release rate for each ejaculation (number of sperm/s).

280 With Spearman's rank correlation analyses we tested whether the ejaculation bout duration 281 correlated with the number of sperm released per ejaculate, and whether the total number of sperm a 282 male released during spawning correlated with the mean number of sperm released per ejaculation, 283 the sperm release rate, and the mean ejaculation bout duration. With Spearman's rank correlation 284 analyses we also checked if the total number of sperm correlates with the number of ejaculations. 285 Furthermore, we tested whether the number of sperm released per ejaculation is constant throughout 286 a spawning during the first 5 hours of spawning. For this purpose, we standardized the data from the eight involved males as follows: [time] = time of ejaculation sample minus time of first sperm release; $[$ sperm per ejaculation $]=$ sperm of ejaculation sample $/$ average sperm per ejaculation. We 289 calculated a linear mixed-effect model (LMM, Bates et al 2014) with time as fixed factor, male ID as random factor and sperm number per ejaculate as dependent variable. The dependent variable was reciprocally transformed as $\frac{1}{\sqrt{\text { sperm per ejaculation+0.5 }}}$. The residuals of the model were checked for normal distribution by visual inspection of the Q-Q plot, and the Kolmogorow Smirnov and 293 Shapiro normality tests. The significance of time was tested using likelihood ratio test between the full model that included time as fixed effect and a null model in which the fixed effect was excluded.

\section{$\underline{\text { Response of nest males to sperm competition with dwarf males }}$}

We checked whether nest males increase their ejaculate investment when faced with a dwarf male experiment, 24 large nest males $(>9 \mathrm{~cm}$; to ensure that they were able to transport shells; Schütz \& 
Taborsky, 2005) were placed individually into 200 litre experimental tanks and given one week of acclimatization time. At the same time, dwarf males were held in a 200 litre storage tank and stimulated with unusable shells and 2 females. Females that had not reproduced for at least 8 weeks were transferred to a 400 litre stimulation tank, in which a nest male was present to stimulate them. When they showed signs of being ready to spawn, two such gravid females were placed in a separate compartment of the experimental tanks (approximately $1 / 4$ of the tank, separated from the nest male by a clear partition) for two days to allow for acclimatisation. A video camera was set in front of the experimental tank to record the nest and surroundings (approximately $1 / 3$ of the experimental tank). Experimental tanks were protected with black curtains from visual disturbance by the observer.

On the day of the experiment, the partition was removed and 3 manipulated shells were put into the tank at 9.00 a.m. The experimental snail shells had a hole $(\mathrm{ca} .0 .5 \times 1 \mathrm{~cm})$ covered with Plexiglas, where normally the dwarf male's body is located during spawning (approximately in the second whorl). This Plexiglas 'window' was fixed to the shell with a rubber band. Tanks were checked every 30 min for courtship activity.

Approximately $3 \mathrm{~h}$ after the first ejaculation of the nest male (i.e., approximately $2 \mathrm{~h}$ after the first egg had been laid; cf. Schütz et al., 2012), we opened the shell's Plexiglas window and added a dwarf male head first into the shell $(\mathrm{N}=12)$. The window was closed again with the rubber band, and the shell was put back into the nest at the same position it had been before handling. If spawning did not start until 12 a.m., the trial was aborted (i.e., the manipulated shells were taken out), and the procedure was started again on the next day to ensure that $9 \mathrm{~h}$ of light were available for the spawning. In the control treatment $(\mathrm{N}=8)$, the procedure was exactly the same (tank equipment, timing, shell handling, camera installation, etc.), except that no dwarf male was added 
into the shell during shell handling.

329 After spawning was finished, the shell with the female was isolated. Shells of the experimental 330 treatment were briefly opened to enable the dwarf male to leave the shell without disturbing the

331 female. The dwarf male was returned to the storage tank. Three days after spawning, the larvae 332 (hatched embryos) were collected by releasing them together with the female with help of light 333 anaesthesia (MS222). Larvae were stored in 95\% ethanol, as were fin clips from nest males, 334 females, and dwarf males.

33615 successfully manipulated spawnings $(\mathrm{N}=7$ of the experimental treatment, $\mathrm{N}=8$ of the control 337 treatment) could be completely video recorded and analysed using Observer 5.0 (Noldus 338 Information Technology). We could not analyse the videos of five spawnings of the experimental 339 treatment because of video system malfunction. We continuously noted the rate of three behaviours 340 known to be of key importance during spawning (Schütz et al., 2012): 'mouthing', when the nest 341 male opens and closes his mouth within the entrance of a shell containing a female; 'ejaculation', 342 when the male stays motionless with his genial papilla over the shell entrance; and 'sniffing', which 343 corresponds to mouthing into an empty shell. Additionally, the duration of each ejaculation bout (as 344 an estimate of sperm release duration), the number of ejaculations, and the time the nest male stayed 345 in his nest were determined over the whole spawning event. The latency from handling until the 346 first ejaculation by the nest male was determined for the experimental and control group to check 347 whether handling affected the two groups differently. A different latency in the experimental 348 treatment compared to the control treatment would indicate different disturbance levels of nest 349 males and females between the two groups. All broods from the experimental treatments (i.e. with a 350 dwarf male contributing to spawning; $\mathrm{N}=12$ ) were tested for paternity using microsatellite analysis 351 (details of DNA sampling and analysis see Appendix). The aim was to analyse at least 20 
354 To analyse the role of the dwarf male presence (treatment: present, control: absent), we subdivided

individuals per brood, if the clutch was large enough. the behavioural recordings into 10-min intervals and calculated means (of rates and durations) per interval for each behavioural variable, starting one $\mathrm{h}$ after the first ejaculation. As with increasing spawning time more and more spawnings end, only the first thirty 10-min intervals were analysed to keep sample size constant (12 intervals before and 18 intervals after handling the shell). From these intervals, we calculated means of behaviours shown before and after the experimental manipulation. With repeated measures ANOVAs, we tested whether the number of ejaculations per female, the ejaculation duration, the time the nest male was in the nest, and the sniffing and mouthing rates differed between before and after the manipulation (within-subjects variables), and whether a difference was due to the treatment effect (between-subjects factor; treatment versus control). We used a Mann-Whitney $U$-test to compare the latency until the first ejaculation by the nest male after handling, between treatment and control conditions. Paternity results were compared with the percentage of total spawning time for which the dwarf male was inside the shell, and with total clutch size.

\section{Ethical note}

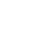

Prior to the laboratory experiments, fish were held in mixed groups of various sizes in tanks between 200 and 500 litres. They were fed twice per day on 6 days of the week as in the experiments. Each individual was used only once for an experiment. After the experiments, fish were placed back into the tanks from which they had been taken. In accordance with the Veterinary office of the Kanton Bern granting permission for our experiments, fish were not anaesthetized for fin clipping in order to reduce stress and potential negative effects of anaesthetization. Fin clips 
were taken from the dorsal and anal fins by removing one fin ray with the surrounding tissue. The

378 removed tissue regrew fully within a period of 4 to 8 weeks. For the field work at Lake Tanganyika, 379 permission was granted by the Fisheries Department of the Ministry of Agriculture and Co380 operatives of Zambia. The laboratory work was licensed by the Veterinärdienst, Amt für 381 Landwirtschaft und Natur des Kantons Bern (license number: 17/09).

\section{Results}

Female visits and sperm competition in nature

On average, 22.31 females visited a nest per day between new moon and one week after full moon (range: 6.7-109.2, Fig. 1). During the same period, the mean number of nest male intruders was 16.14 per day and nest (range: 3.6-26.0; mainly neighbouring nest males that were expelled by the nest owner), whereas the corresponding number of sneakers entering the nest was on average 0.83 (range: 0-5.4) and that of dwarf males 0.38 (range: 0-2.14, Fig. 1).

During the moon cycle, which was separated into seven 3-day periods, the frequencies of visiting 394 females $\left(X_{6}=16.74, P=0.010\right)$ and of reproductive parasites (dwarf and sneaker males combined) differed significantly from an equal distribution $\left(X_{6}=20.871, P=0.002\right)$, but the frequency of nest male intrusions did not $\left(X_{6}=8.27, P=0.205\right)$. Furthermore, the distribution of visiting females differed significantly from the distribution of parasitic males attempting to participate in spawning $\left(X_{6}=15.597, P=0.016\right)$ and from the distribution of nest male intrusions $\left(X_{6}=21.5, P=0.0015\right)$. 
Nest males were spawning with up to four females simultaneously (28 spawnings corresponding to 28 different females, recorded in 10 monitored nests). The total spawning duration of a clutch significantly depended on the number of females spawning at a time (GLM, $F_{1,3}=4.536, P=$ $0.015)$, whereas it was not related to male identity $(\mathrm{df}=8, \mathrm{~F}=1.265, \mathrm{p}=0.321)$. The number of ejaculations per female neither seemed to be influenced by the number of simultaneously spawning females $\left(\mathrm{GLM}, F_{1,3}=0.811, P=0.505\right)$ nor by male identity $\left(F_{1,8}=1.296, P=0.310\right)$. However, viewed on a continuous scale, the number of ejaculations per female tended to decline with increasing numbers of simultaneously spawning females (Spearman rank correlation, $r_{S}=-0.353, N$ $=28, P=0.065$, see Fig. 2).

\section{$\underline{\text { Sperm release rate versus the duration of ejaculations }}$}

Both the average number of sperm per ejaculation (Spearman rank correlation, $r_{S}=0.905, N=8, P$ $=0.002$, Fig. $3 \mathrm{a}$ ) and the sperm release rate (sperm number per ejaculate / duration of ejaculation bout in s, Spearman rank correlation, $r_{S}=0.881, N=8, P=0.004$, Fig. $3 b$ ) correlated positively with the total number of sperm a male released during spawning. The mean duration of ejaculation bouts related neither to the total number of sperm (Spearman rank correlation, $r_{S}=0.143, N=8, P=$ 0.736 ) nor to the number of sperm released per ejaculate (Spearman's rank correlation, $r_{S}=-0.024$, $N=8, P=0.955$, Fig. $3 c)$.

\section{The number of ejaculations serves as a good proxy for the total number of sperm released since (i)} there is a positive correlation between the number of ejaculations and the total number of sperm released (Spearman rank correlation, $r_{S}=0.857, N=8, P=0.007$ ) and (ii) the number of sperm per ejaculation remains rather constant over the majority of spawning time. During the first 5 hours of spawning, time showed no significant effect on the number of sperm per ejaculate (model 
427 comparison with likelihood ratio test of the two models with and without time as fixed factor, Chi ${ }^{2}$ $428=0.742, \mathrm{df}=1, \mathrm{p}=0.389)$.

$430 \quad$ Response of nest males to sperm competition with dwarf males

Nest male behaviour

Repeated measures ANOVAs determined that the ejaculation duration and the number of ejaculations per female differed before and after adding a dwarf male into the shell, and that this difference was due to the treatment (comparing treatment with control conditions; table 1, Fig. 4). After experimentally adding a dwarf male, the duration of ejaculation bouts per female dropped by $61.5 \%$ and the number of ejaculations per female declined by $40.5 \%$ in comparison to the experimental control (Fig. 4, right panels, A: dwarf versus control). The mouthing and sniffing rates, and the time the nest male stayed inside the nest also differed between before and after the 441 shell manipulation, but this was not explained by the treatment (table 1). The latency to the first 442 ejaculation occurring after handling did not differ between the treatment and control groups (Mann443 Whitney U test: $\left.U=25, N_{1}=7, N_{2}=8, P=0.779\right)$, suggesting that handling per se did not affect 444 the two experimental groups differently.

Paternity

448 The percentage of larvae the dwarf male sired correlated positively with the percentage of total 449 spawning time for which it had been in the shell during spawning (Spearman rank correlation, $r_{S}=$ $4500.867, N=7, P=0.012$ ), whereas it did not correlate with the clutch size (Spearman rank 451 correlation, $\left.r_{S}=-0.078, N=12, P=0.809\right)$. Microsatellite analyses showed that dwarf male 
paternity ranged between zero and 100 percent $\left(x ;^{-}=13.6 \%\right.$; see Appendix, table A1). In 6 of 12 cases the dwarf male did not fertilize any eggs.

\section{Discussion}

Long-term video surveys of L. callipterus nests in the field revealed that many neighbouring nest males intrude into a territory during spawning, mainly trying to steal shells or fertilisations (Maan \& Taborsky, 2008). In addition, sneaker and dwarf males enter the nests during spawning. Although sneaker males occasionally succeed in releasing sperm into a shell where a female is laying eggs, their fertilisation success is very low in comparison to dwarf males (Wirtz Ocana et al., 2014), because due to the time pattern of egg release (Schütz et al., 2012) they can fertilise only one egg per ejaculation. Thus, whereas dwarf males outcompete nest males in fertilisations, sperm competition with sneaker males is of minor importance (Wirtz Ocana et al., 2014). The distributions of intruding males and nest visiting females differed from each other during the moon cycle.

Therefore, nest males face different levels of sperm competition during their territory holding period, which should select for an ability to adjust ejaculation characteristics to the current risk of sperm competition.

The total spawning duration a nest male spent for each clutch declined with an increasing number of simultaneously spawning females, whereas male identity had no obvious effect. Additionally, the number of ejaculations per spawning tended to decrease with increasing numbers of simultaneously spawning females. Since the number of ejaculations is positively correlated with the total number of sperm released, and the number of sperm per ejaculation remains constant over the majority of spawning time (see also Schütz et al., 2010), the ejaculation number per female is a good predictor for the number of sperm released per female. These changes in spawning behaviour likely reflect 
sperm depletion in males, which was revealed also when nest males spawned with single females

for longer periods than five h (Schütz et al., 2010). Sperm depletion of males may lead to a decrease in female productivity (Dunn, Andrews, Ingrey, Riley, \& Wedell, 2006) and to sexual conflict over the size and distribution of ejaculates (Diaz, Haydon, \& Lindstrom, 2010; Smith, Pateman-Jones, Zieba, Przybylski, \& Reichard, 2009). Female choice may hence be influenced by potential sperm limitation of males. In L. callipterus, females may reduce the risk of suffering from nest male sperm limitation by choosing large nest owners (Maan \& Taborsky, 2008), and by enabling dwarf males to enter the shell during later phases of spawning, when nest males are already sperm depleted.

Our laboratory experiments revealed that the mean number of sperm released per ejaculate and the sperm release rate were good predictors for the total number of sperm released for a whole clutch. However, the mean duration of ejaculation bouts did not significantly correlate with the number of sperm released per ejaculation or with the total number of sperm released per spawning. This might suggest that our estimate of the actual ejaculation duration as derived from the amount of time the male stays motionless with his genial papilla over the shell entrance may be inaccurate. Nest males may not release sperm during the whole time holding this position, and therefore the number of ejaculations per female is a better measure for male sperm expenditure than ejaculation bout duration.

Males seem to increase the number of sperm released during a spawning mostly through a higher sperm release speed rather than by increasing the duration of ejaculation bouts. This can be explained by the peculiar spawning condition in L. callipterus, where each egg has to be fertilised by a separate ejaculate of the nest male over a period of many hours (Schütz et al., 2012). In principle, males have three possibilities to raise the total sperm number and to increase their fertilisation probability. They may increase the ejaculation frequency, the duration of ejaculation 
bouts, or the sperm release rate. However, since females obviously determine the timing of egg laying (adopting quite regular intervals of 2 minutes between subsequent eggs; Schütz et al., 2012), nest males have to assume this pattern of gamete release. Thus, nest males would not enhance fertilization chances by increasing the ejaculation frequency or the duration of ejaculation bouts, hence raising the sperm release rate per ejaculate seems to be their best option (i.e. per egg to be fertilized).

Inducing sperm competition experimentally showed that nest males did not increase their sperm expenditure as predicted by sperm competition risk models (Parker, 1990b; Parker, 1998), but rather decreased it as predicted by sperm competition intensity theory (Parker et al., 1996; Parker et al., 1997; Wedell et al., 2002). Adding a dwarf male into a shell when a nest male was spawning resulted in $61.5 \%$ shorter ejaculation bout durations of the nest male, and in $40.5 \%$ fewer ejaculations per spawning female than in the control experiment. Apparently, nest males decrease their ejaculate expenditure on average by roughly $50 \%$ when dwarf males are present to save sperm for better future spawning opportunities. The mouthing and sniffing rates and the time the nest male stayed inside the nest also differed between the periods before and after the manipulation, but these differences were not explained by the treatment effect, so they reflected rather a response to the manipulation.

Modulation of ejaculate investment by which males increase the sperm number released when sperm competition risk is high has been observed in several species with internal fertilization and sperm storage (Wedell et al., 2002). For example, with increasing perceived risk of sperm competition, males increased the amount of sperm transferred in two cricket species, Acheta domesticus and Gryllodes supplicans (Gage \& Barnard, 1996), in Drosophila melanogaster (Moatt, Dytham, \& Thom, 2014), in the South American fruit fly Anastrepha fraterculus (Abraham, Teresa, 
\& Perez-Staples, 2015), and in the lekking lesser wax moth, Achroia grisella (Jarrige, Riemann,

528 Goubault, \& Schmoll, 2015). In horses, Equus caballus, stallions experiencing a high risk of sperm

529 competition ejaculated more sperm after exposure to mares that had previous contact to other

530 stallions than males that experienced a low risk of sperm competition (no previous exposure to

531 other stallions; Burger, Dolivo, \& Wedekind, 2015).

533 Regarding species with a fertilization mode similar to that of L. callipterus, ejaculation

534 characteristics have been shown to be adjusted to the presence of rival males in two species of

535 bitterlings. These fish are spawning into the gill chambers of living mussels with fertilization taking

536 place inside the gill cavity, resembling a female reproductive tract in internally fertilizing species

537 (Smith, Warren, Rouchet, \& Reichard, 2014). When faced with rival male ejaculations, male

538 Chinese rose bitterling, Rhodeus ocellatus, decreased the overall ejaculation rate, but released

539 sperm into the mussels more frequently (Smith et al., 2014). In contrast to L. callipterus, territorial

540 male European bitterling, Rhodeus sericeus, increased ejaculate expenditure when competing with a

541 rival male in comparison to situations when spawning alone (Smith, Reichard, \& Jurajda, 2003).

542 They decreased ejaculate expenditure, however, with an increasing numbers of competitors (Smith

543 et al., 2003), confirming two predictions of sperm competition risk and intensity models (Parker,

544 1990b; Stockley et al., 1997). In contrast to parasitic dwarf males in L. callipterus, which are in a

545 superior spawning position inside the shell, in bitterlings it has been assumed that sperm

546 competition between guarders and sneakers resembles a fair raffle, where each male fertilizes a

547 number of eggs proportional to his contribution of sperm (Smith \& Reichard, 2013). We assume

548 that due to the unprivileged position of L. callipterus nest males when spawning with dwarf male

549 participation (Wirtz Ocana et al., 2014), nest males are unable to compensate for offspring loss to

550 dwarf males efficiently by increasing sperm numbers. Thus, when spawning with parasitic males

551 present inside the shell, due to the highly loaded raffle (Parker et al., 1997) in favour of the parasite 
552

it seems advantageous for nest males to conserve sperm for better spawning opportunities in the future, without dwarf male participation.

Paternity analysis showed that the proportion of offspring sired by the dwarf male correlated highly with the percentage of spawning time the latter was inside the shell, whereas it did not relate to clutch size. In our lab experiment the relative reproductive success of dwarf males was considerably smaller than dwarf male success in the natural situation (Wirtz Ocana et al., 2014). In half of the 12 broods where a dwarf male was experimentally added into the shell they did not sire any offspring, and the average percentage of larvae a dwarf male sired was $13.6 \%$. In contrast, all of 10 broods with dwarf male participation collected in the field contained dwarf male offspring (range: 15.6$100 \%)$, and the majority of offspring were sired by the dwarf male in those cases $\left(x ;{ }^{-}=77.6 \%\right.$, Wirtz Ocana et al., 2014). This difference in dwarf male success between field and experimental lab situation was probably due to the influence of our manipulation, by which dwarf males were forced into their position inside the shell. In nature, successful wriggling into a shell and dwarf male participation in spawning depend on the relative sizes of the dwarf male, the female and the shell (Sato et al., 2004). To participate in spawning in our laboratory experiment, dwarf males had no choice between shells or females, thus important parameters might not have been appropriate for them to behave normally. However, our data show that nest males responded strongly to the presence of dwarf males in the shell during spawning, which enabled us to scrutinize this response with respect to adjustments in their ejaculate release.

Earlier studies showed that the average spawning duration was $6.9 \mathrm{~h}$ in the field (Schütz et al., 2012). However, the number of sperm released per ejaculate of nest males dropped sharply by $88 \%$ on average after $5 \mathrm{~h}$ of spawning, even when males were spawning with only one female at a time (Schütz et al., 2010), which suggests sperm depletion. If nest males are limited to fertilize eggs laid 
during the last $1.9 \mathrm{~h}$ of spawning, they might loose up to $27.5 \%(1.9 / 6.9)$ of offspring per brood due to sperm limitation, and probably considerably more when spawning with several females simultaneously. Our study showed that nest males decrease ejaculate expenditure by roughly $50 \%$ when dwarf males are present. Since nest males sired on average only $22.4 \%$ of offspring when dwarf males succeeded to enter a shell in nature (Wirtz Ocana et al., 2014), they might do better by saving sperm when competing with a rival in a highly disadvantaged position. In spawnings without dwarf male participation, nest males sire virtually all offspring (Wirtz Ocana et al., 2014). Our study showed that in the field, nest males spawned with only one female at a time in $62.9 \%$ of all cases, simultaneously with two females in $14.3 \%$ of cases, and with 3 and 4 females in $11.4 \%$ of cases each. Thus, the probability to spawn with more than one female at a time corresponds to about $37 \%$, which substantially increases the possibility to make use of sperm saved from spawnings with dwarf male participation.

To our knowledge, this is the first study showing that ejaculate expenditure decreases in the presence of a single rival male in externally fertilising fish. When a parasitic dwarf male participates in spawning, nest males reduce ejaculate expenditure by roughly $50 \%$, which is contrary to predictions from ESS models of responses to sperm competition risk (Parker, 1990a; Parker, 1998). Apparently, nest males save sperm for future mating opportunities as predicted by sperm competition intensity models (Parker, Ball, Stockley, \& Gage, 1996; 1997). Results from this and previous studies combined suggest that this is due to the unprivileged position of L. callipterus nest males when spawning with parasitic dwarf males, which differs from the competition between bourgeois and parasitic males during spawning in most other species. Nest males are highly sperm limited even when spawning with only one female, and their ejaculate expenditure decreases significantly when spawning with more than two females simultaneously. Obviously, in $L$. callipterus nest males the proposed trade-off between investing in the current or in future matings 
602 (Wedell et al., 2002) is solved by reducing ejaculate expenditure when in competition with superior 603 dwarf males and thereby conserving sperm for future spawning opportunities without dwarf males. 604 605 Acknowledgements 606

We thank the Fisheries Department of the Ministry of Agriculture and Co-operatives of Zambia for 608 the permission to study the fish at Lake Tanganyika, Danielle Bonfils for performing the paternity 609 analyses, and Leif Engqvist and Barbara Taborsky for help with statistics. This work was funded by 610 the Swiss National Science Foundation (grant number 310030B_138660 to MT).

\section{References}

614 Abraham, S., Teresa V. M., \& Perez-Staples, D. (2015). Current sperm competition determines sperm allocation in a tephritid fruit fly. Ethology, 121, 451-461.

616 Bates, D. M., Mächler, M., Bolker, B. M., \& Walker S. C. (2014). lme4: Linear Mixed-Effects Models Using Eigen and S4. R Package Version 1.1-7. Available online at: http://CRAN.Rproject.org/package $=$ lme4

Birkhead, T. R. (1998). Sperm competition in birds. Reviews of Reproduction, 3, 123-129.

Burger, D., Dolivo, G., \& Wedekind, C. (2015). Ejaculate characteristics depend on social environment in the horse (Equus caballus). Plos One, 10 (11), p. e0143185.

Byrne, P. G., Roberts, J. D., \& Simmons, L. W. (2002). Sperm competition selects for increased testes mass in Australian frogs. Journal of Evolutionary Biology, 15, 347-355.

Diaz, S. A., Haydon, D. T., \& Lindstrom, J. (2010). Sperm-limited fecundity and polyandryinduced mortality in female nematodes Caenorhabditis remanei. Biological Journal of the Linnean Society, 99, 362-369. 
Dunham, A., \& Rudolf, V. (2009). Evolution of sexual size monomorphism: the influence of passive mate guarding. Journal of Evolutionary Biology, 22, 1376-1386.

Dunn, A. M., Andrews, T., Ingrey, H., Riley, J., \& Wedell, N. (2006). Strategic sperm allocation under parasitic sex-ratio distortion. Biology Letters, 2, 78-80.

Engqvist, L., \& Reinhold, K. (2005). Pitfalls in experiments testing predictions from sperm competition theory. Journal of Evolutionary Biology, 18, 116-123.

Jarrige, A., Riemann, D., Goubault, M., \& Schmoll, T. (2015). Strategic sperm allocation in response to perceived sperm competition risk in a lekking insect. Animal Behaviour, 109, 8187.

Kelly, C. D., \& Jennions, M. D. (2011). Sexual selection and sperm quantity: meta-analyses of strategic ejaculation. Biological Reviews, 86, 863-884.

Leong, R. J. H. (1989). Sperm concentrations and egg fertilization rates during spawning of captive anchovy, Engraulis mordax. California Cooperative Oceanic Fisheries Investigations, 30, 136139.

Levitan, D. R. (2002). Density-dependent selection on gamete traits in three congeneric sea urchins. Ecology, 83, 464-479.

Mitchell, J., Wirtz Ocana, S., \& Taborsky, M. (2014). Male and female shell-brooding cichlids prefer different shell characteristics. Animal Behaviour, 98, 131-137.

Maan, M. E., \& Taborsky, M. (2008). Sexual conflict over breeding substrate causes female expulsion and offspring loss in a cichlid fish. Behavioural Ecology, (19, 302-308.

Moatt, J. P., Dytham, C., \& Thom, M. D. (2014). Sperm production responds to perceived sperm competition risk in male Drosophila melanogaster. Physiology \& Behavior, 131, 111-114.

Nakai, K., Yanagisawa, Y., Sato, T., Niimura, Y., \& Gashagaza, M. M. (1990). Lunar synchronisation of spawning in cichlid fishes of the tribe Lamprologini in Lake Tanganyika. Journal of Fish Biology, 37, 589-598. 
652 Oliveira, R. F., Taborsky, M., \& Brockmann, H. J. (2008). Alternative Reproductive Tactics. (1 653 edn.). Cambridge: Cambridge University Press.

654 Parker, G. A. (1970). Sperm competition and its evolutionary consequences in the insects. $655 \quad$ Biological Review, 45, 525-567.

656 Parker, G. A. (1990a). Sperm competition games: raffles and roles. Proceedings of the Royal 657 Society of London Series B, 242, 120-126.

658 Parker, G. A. (1990b). Sperm competition games: sneaks and extra-pair copulations. Proceedings of 659 the Royal Society of London Series B, 242, 127-133.

660 Parker, G. A. (1998). Sperm competition and the evolution of ejaculates: towards a theory base. In: 661 T. Birkhead, \& A. P. Møller (Eds.), Sperm Competition and Sexual Selection (pp. 3-54). San 662 Diego, London: Academic press.

663 Parker, G. A., \& Simmons, L. W. (1994). Evolution of phenotypic optima and copula duration in 664 dungflies. Nature, 370, 53-56.

665 Parker, G. A., Ball, M. A., Stockley, P., \& Gage, M. J. G. (1996). Sperm competition games: 666 assessment of sperm competition intensity by group spawners. Proceedings of the Royal 667 Society of London Series B, 263, 1291-1297.

668 Parker, G. A., Ball, M. A., Stockley, P., \& Gage, M. J. G. (1997). Sperm competition games: a 669 prospective analysis of risk assessment. Proceedings of the Royal Society of London Series B, $670 \quad 264,1793-1802$.

671 Pateman-Jones, C., Rasotto, M. B., Reichard, M., Liao, C., Liu, H., Zieba, G., \& Smith, C. (2011). 672 Variation in male reproductive traits among three bitterling fishes (Acheilognathinae: 673 Cyprinidae) in relation to the mating system. Biological Journal of the Linnean Society, 103, $674 \quad 622-632$.

675 R Core Team (2015) R: a language and environment for statistical computing, Vienna, Austria, 676 https:Iwww.r-project.org/ 
Reichard, M., Ondrackova, M., Bryjova, A., Smith, C., \& Bryja, J. (2009). Breeding resource ristribution affects selection gradients on male phenotypic traits: Experimental study on lifetime reproductive success in the bitterling fish (Rhodeus amarus). Evolution, 63, 377-390.

Sato, T. (1994). Active accumulation of spawning substrate: a determinant of extreme polygyny in a shell-brooding cichlid. Animal Behaviour, 48, 669-678.

Sato, T., Hirose, M., Taborsky, M., \& Kimura, S. (2004). Size-dependent male alternative reproductive tactics in the shell-brooding cichlid fish Lamprologus callipterus in Lake Tanganyika. Ethology, 110, 49-62.

Schöfl, G., \& Taborsky, M. (2002). Prolonged tandem formation in firebugs (Pyrrhocoris apterus) serves mate-guarding. Behavioral Ecology and Sociobiology, 52, 426-433.

Schütz, D., Heg-Bachar, Z., Taborsky, M., \& Heg, D. (2012). Spawning coordination of mates in a shell brooding cichlid. International Journal of Evolutionary Biology, Volume (2012, 1-10. Article ID: 517849.

Schütz, D., Pachler, G., Ripmeester, E., Goffinet, O., \& Taborsky, M. (2010). Reproductive investment of giants and dwarfs: Specialized tactics in a cichlid fish with alternative male morphs. Functional Ecology, 24, 131-140.

Schütz, D., Parker, G. A., Taborsky, M., \& Sato, T. (2006). An Optimality approach to male and female body sizes in an extremely size dimorphic cichlid fish. Evolutionary Ecology Research, 8, 1393-1408.

Schütz, D., \& Taborsky, M. (2000). Giant males or dwarf females: what determines the extreme sexual size dimorphism in Lamprologus callipterus? Journal of Fish Biology, 57, 1254-1265.

Schütz, D., \& Taborsky, M. (2005). The influence of sexual selection and ecological constraints on an extreme sexual size dimorphism in a cichlid. Animal Behaviour, 70, 539-549.

Shapiro, D. Y., \& Giraldeau, L. A. (1996). Mating tactics in external fertilizers when sperm is limited. Behavioral Ecology, 7, (19-23. 
Shapiro, D. Y., Marconato, A., \& Yoshikawa, T. (1994). Sperm economy in a coral-reef fish, Thalassemia bifasciatum. Ecology, 75, 1334-1344.

Smith, C., Pateman-Jones, C., Zieba, G., Przybylski, M., \& Reichard, M. (2009). Sperm depletion as a consequence of increased sperm competition risk in the European bitterling, Rhodeus amarus. Animal Behaviour, 77, 1227-1233.

Smith, C., \& Reichard, M. (2013). A sperm competition model for the European bitterling (Rhodeus amarus). Behaviour, 150, 1709-1730.

Smith, C., Reichard, M., \& Jurajda, P. (2003). Assessment of sperm competition by European bitterling, Rhodeus sericeus. Behavioral Ecology and Sociobiology, 53, (206-213.

Smith, C., Warren, M., Rouchet, R., \& Reichard, M. (2014). The function of multiple ejaculations in bitterling. Journal of Evolutionary Biology, 27, 1819-1829.

Snow, L. S. E., Abdel-Mesih, A., \& Andrade, M. C. B. (2006). Broken copulatory organs are lowcost adaptations to sperm competition in redback spiders. Ethology, 112, 379-389.

Stockley, P., Gage, M. J. G., Parker, G. A., \& Møller, A. P. (1997). Sperm competition in fishes: the evolution of testis size and ejaculate characteristics. American Naturalist, 149, 933-954.

Taborsky, M. (1997). Bourgeois and parasitic tactics: do we need collective, functional terms for alternative reproductive behaviours? Behavioral Ecology and Sociobiology, 41, 361-362.

Taborsky, M. (1998). Sperm competition in fish: 'bourgeois' males and parasitic spawning. Trends in Ecology and Evolution, 13, 222-227.

Taborsky, M. (2001). The evolution of bourgeois, parasitic, and cooperative reproductive behaviors in fishes. Journal of Heredity, 92, 100-110.

Taborsky, M. (2008). Alternative reproductive tactics in fish. In R. Oliveira, M. Taborsky, \& H. J. Brockmann (Eds.), Alternative Reproductive Tactics: An Integrative Approach (pp. 251-299). Cambridge: Cambridge University Press.

Taborsky, M., Oliveira, R. F., \& Brockmann, H. J. (2008). The evolution of alternative reproductive 
tactics: concepts and questions. In R. F. Oliveira, M. Taborsky, \& H. J. Brockmann (Eds.), Alternative Reproductive Tactics: An Integrative Approach (pp. 1-21). Cambridge: Cambridge University Press.

730 Taborsky, M. \& Brockmann, H. J. (2010). Alternative tactics and life history phenotypes. In P. M. Kappeler (Ed.), Animal Behaviour: Evolution and mechanisms (pp. 537-586). Berlin: Springer Verlag.

733 Wedell, N., Gage, M. J. G., \& Parker, G. A. (2002). Sperm competition, male prudence and sperm$734 \quad$ limited females. Trends in Ecology \& Evolution, 17, 313-320.

735 White, D., Braeden, B., Creswell, D., \& Smith, C. (1998). MagenSil ${ }^{\mathrm{TM}}$ Paramagnetic Particles: 736 Novel Magnetics for DNA Purification. Promega Notes, 69.

737 Wirtz Ocana, S., Meidl, P., Bonfils, D., \& Taborsky, M. (2014). Y-linked Mendelian inheritance of 738 giant and dwarf male morphs in shell-brooding cichlids. Proceedings of the Royal Society of $739 \quad$ London Series B, 281, 20140253. 


\section{Appendix: Details of the paternity analysis}

742 Genomic DNA was extracted from ethanol-preserved fin clip samples (1-2 $\mathrm{mm}^{2}$ each) and whole 743 larvae with a manual 96-well format DNA extraction protocol on the basis of a magnetic separation 744 technique (White, Braeden, Creswell, \& Smith, 1998). According to the protocol of the Wizard® 745 Genomic DNA Purification Kit (Technical Manual No. TM050; Promega), tissue lysis was 746 performed in a Lysis-Buffer consisting of Nuclei Lysis Solution (Promega), 0.5M EDTA (Sigma747 Aldrich) and Proteinase K (Qiagen AG). By adding MagneSil® Paramagnetic Particles (Promega; 748 (White et al. 1998) to the lysate, DNA was captured in solution and washed 2-3 times with 80\% 749 ethanol with the aid of a magnetic separator (MagnaBot®96 Magnetic Separation Device, Promega, 750 Cat.\# V8151) to eliminate residual contaminations. DNA was finally eluted in 50-100 $\mu$ l of distilled 751 water.

Eleven microsatellite primer pairs (loci NP007, NP773, ULI2, Pzeb3, Pzeb4, TmoM5, TmoM13, TmoM25, TmoM27, UME003 and UNH154; see Wirtz Ocana et al. 2014) were multiplexed in one polymerase chain reaction using the QIAGEN Multiplex PCR Kit (Qiagen). PCR reactions were carried out in a $10 \mu \mathrm{l}$ volume containing 1-2 $\mu$ l of the genomic DNA, 1x QIAGEN Multiplex PCR Master Mix (consisting of QIAGEN Multiplex PCR buffer with a final concentration of $3 \mathrm{mM}$ $\mathrm{MgCl}_{2}, \mathrm{dNTP}$ mix, and HotStarTaq DNA polymerase), $0.1 \mu \mathrm{M}$ of locus-specific $5^{\prime}$ fluorescentlabelled forward primer [fluorescent dyes: 6-FAM, HEX (Microsynth), NED and PET (Applied Biosystems)], and non-labelled reverse primers.

762 Amplification was done in a 96-well GeneAmp® PCR System 9700 (Applied Biosystems) using 763 the following cycling protocol: $15 \mathrm{~min}$ at $95^{\circ} \mathrm{C} ; 35$ cycles consisting of $30 \mathrm{~s}$ at $95^{\circ} \mathrm{C}, 3 \mathrm{~min}$ at $57^{\circ} \mathrm{C}$, 764 and $1 \mathrm{~min}$ at $72^{\circ} \mathrm{C}$, followed by a final 15 -min extension at $72^{\circ} \mathrm{C}$. Fluorescent PCR fragments were 
765 visualised by capillary electrophoresis on an ABI PRISM® 3100 Genetic Analyzer. Genotypes

766 were scored automatically using GeneMapper software version 3.7 (Applied Biosystems) against an

767 internal size standard (GeneScan 500 LIZ, Applied Biosystems) and revised manually to ensure 768 genotyping consistency.

769

770 Since the present experimental groups consisted of breeding females with their offspring and only 2

771 potential fathers ( 1 nest male and 1 dwarf male), maternity was always certain, and therefore

772 assignment of paternity was very simple, performed visually via allelic matching at all 11 loci. For

773 all of the offspring tested, parentage could be assigned unambiguously, there were 0 mismatching

774 alleles with the respective parents.

775 
778 Table 1: Differences in behaviours before and after manipulation of a shell with a spawning female 779 inside, and treatment effect (experimental addition of a dwarf male versus control manipulation): 780 Results of repeated measures ANOVAs. Significant differences are highlighted in bold. 781

\begin{tabular}{lcccc}
\hline & \multicolumn{2}{c}{ Variable } & \multicolumn{2}{c}{ Variable $\times$ treatment } \\
& $F_{1,13}$ & $P$ & $F_{1,13}$ & $P$ \\
\hline Ejaculation duration & 12.203 & $\mathbf{0 . 0 0 4}$ & 6.67 & $\mathbf{0 . 0 2 3}$ \\
Ejaculations / female & 38.129 & $\mathbf{0 . 0 0 1}$ & 7.809 & $\mathbf{0 . 0 1 5}$ \\
Mouthing & 22.677 & $\mathbf{0 . 0 0 1}$ & 0.004 & 0.95 \\
Sniffing & 5.366 & $\mathbf{0 . 0 3 7}$ & 1.81 & 0.202 \\
Time in nest & 7.401 & $\mathbf{0 . 0 1 7}$ & 3.075 & 0.103 \\
& & & & \\
\hline
\end{tabular}




\section{Acknowledgements}

We thank the Fisheries Department of the Ministry of Agriculture and Co-operatives of Zambia for the permission to study the fish at Lake Tanganyika, Danielle Bonfils for performing the paternity analyses, and Leif Engqvist and Barbara Taborsky for help with statistics. This work was funded by the Swiss National Science Foundation (grant number $\underline{310030 \mathrm{~B} \_138660 \text { to MT)} .}$ 
Figure 1

Figure 1
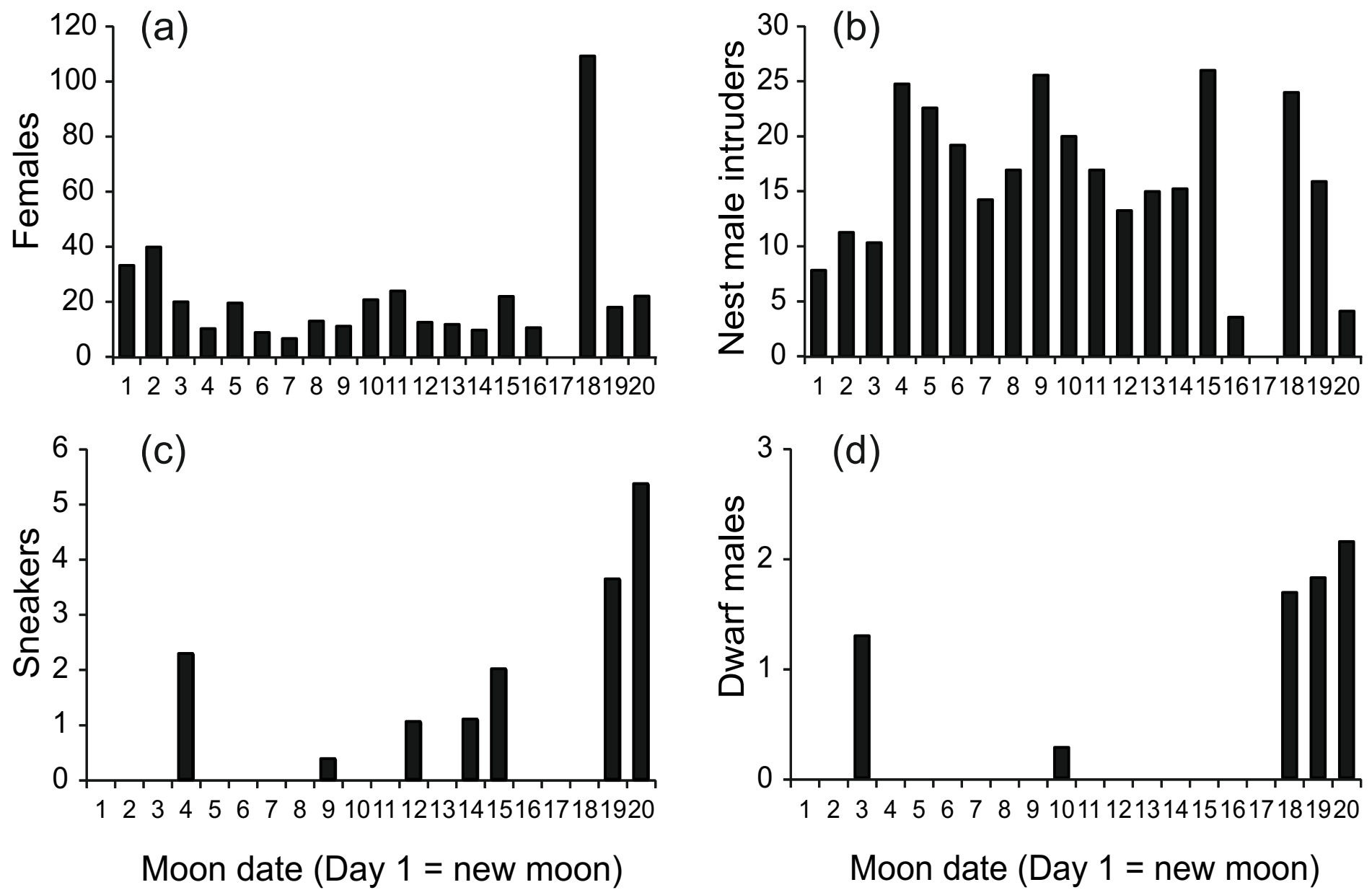

Moon date (Day $1=$ new moon)

Moon date (Day 1 = new moon) 
Figure 2

Figure 2
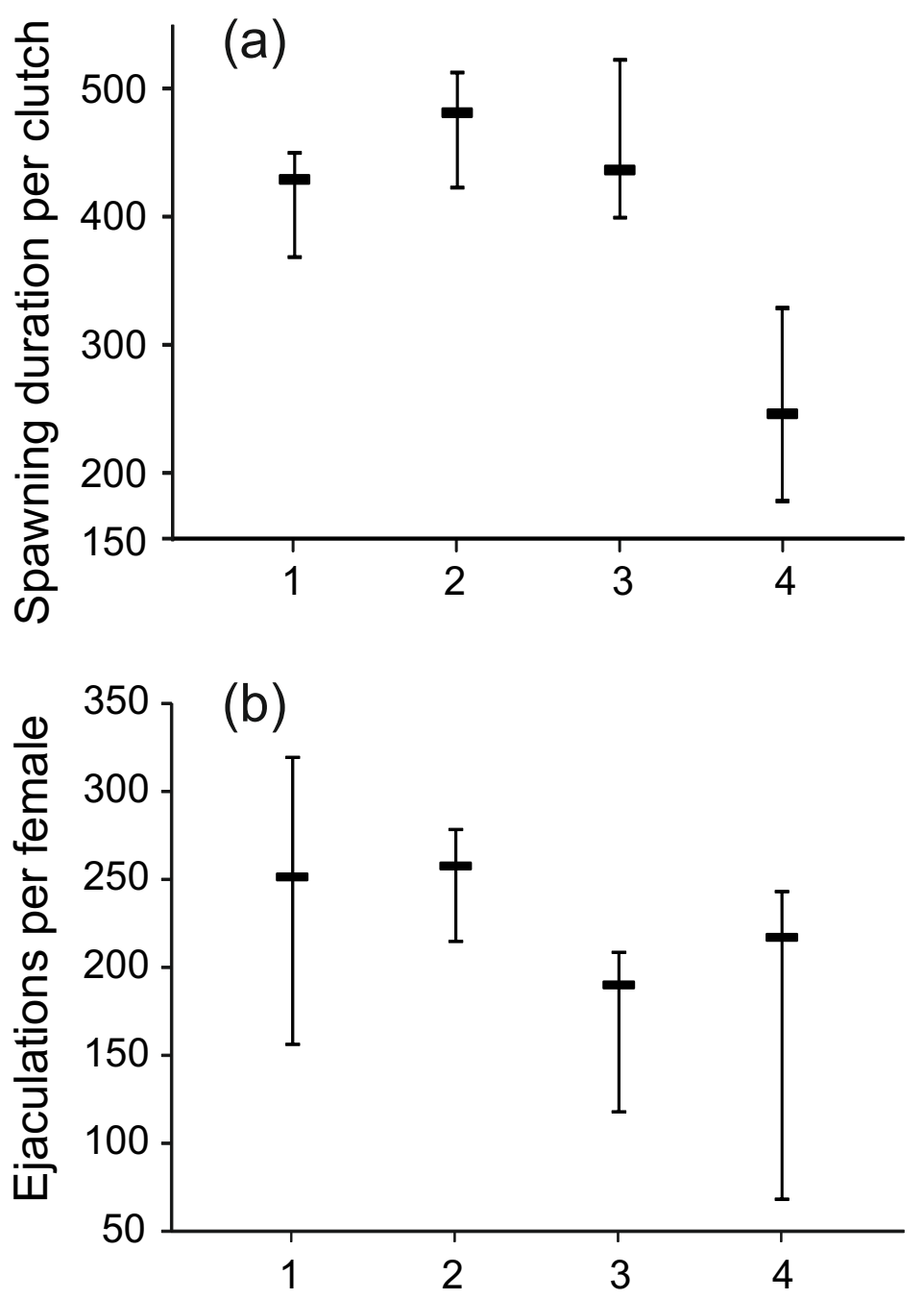

Simultaneously spawning females 
Figure 3
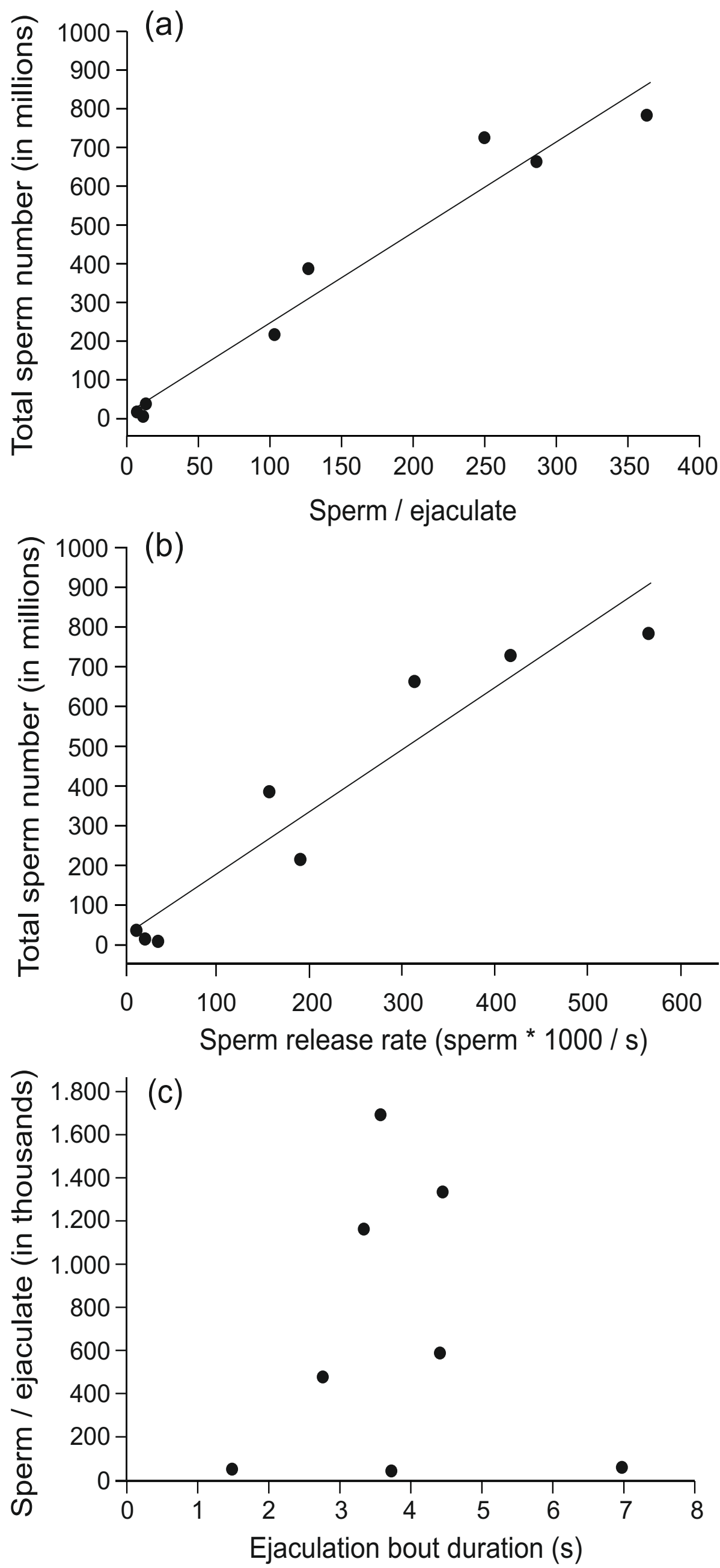
Figure 4
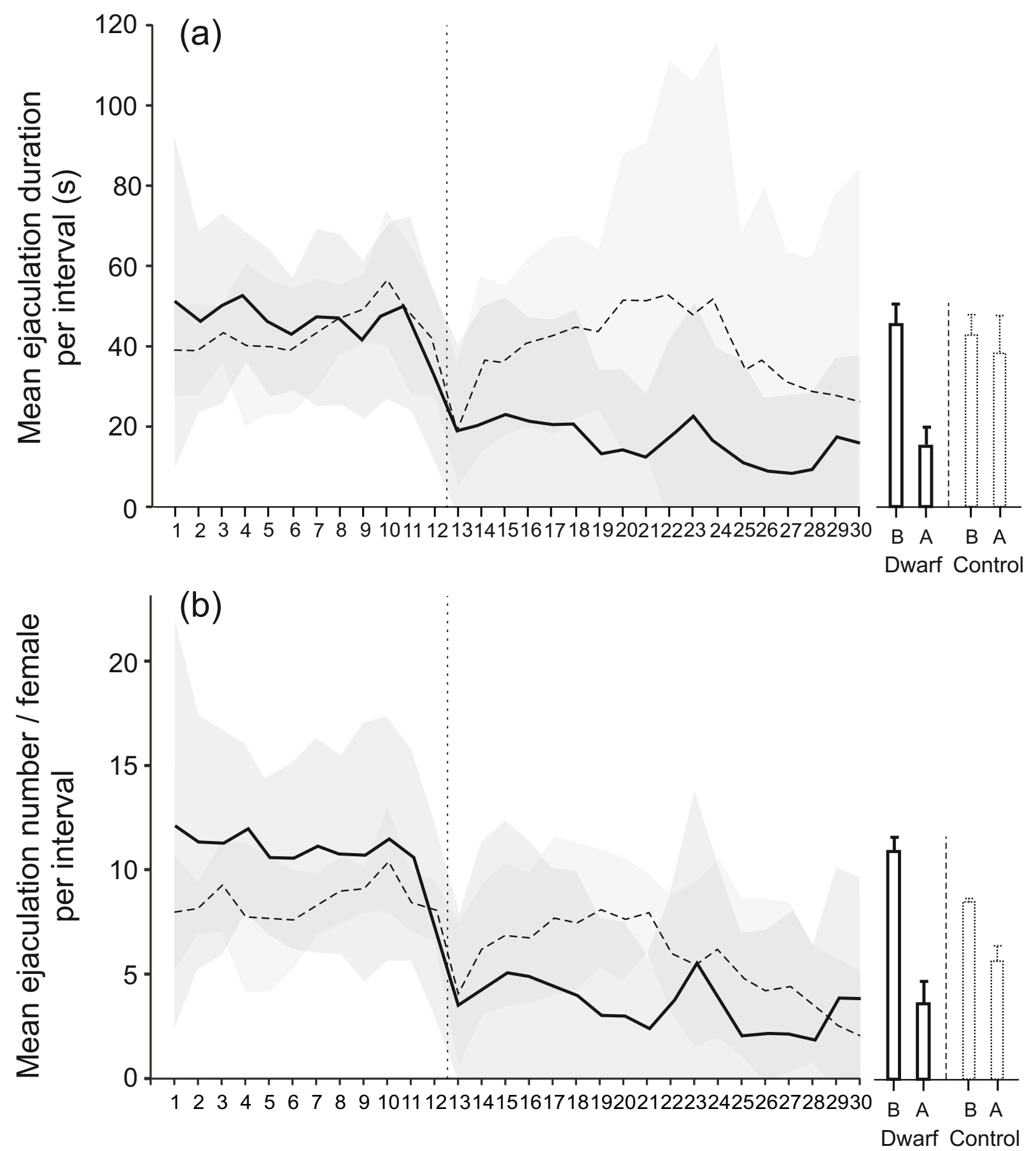